\title{
A low-cost interface for multi-electrode array data acquisition systems
}

\author{
Michael Serra, Amy Chan, Maya Dubey, and Thomas B. Shea \\ Center for Cellular Neurobiology and Neurodegeneration Research, \\ University of Massachusetts, Lowell, MA, USA
}

BioTechniques 45:451-456 (October 2008)

doi $10.2144 / 000112911$

\begin{abstract}
Multi-electrode array systems have enabled the in vitro electrophysiological study of neuronal networks. The data processing component of these systems consists of an advanced computer system and data acquisition electronics that collectively cost more than the multi-electrode arrays and amplifiers. Considering that these elaborate systems may be cost-prohibitive for many laboratories, we have developed a simple but novel method for recording groups of related multi-electrode array channels with a low-cost data acquisition system.
\end{abstract}

Multi-electrode array (MEA) systems enable the analysis of neuronal network activity in vitro, and consist of an electrode array embedded within a cell culture dish (Figure 1). Electrical potentials produced by a neuronal network are detected, amplified, and recorded; and can be analyzed by a variety of computer programs $(1,2)$.

The total cost of an MEA system (\$30,000-\$50,000; MultiChannel Systems, Reutlingen, Germany) precludes its availability to many laboratories. The least expensive amplifier is $\sim \$ 10,000$ (MEA1060-INV; Multichannel Systems), and data acquisition systems that record 60 channels at 50 $\mathrm{kHz}$ each cost $>\$ 35,000$ (MC Card; Multichannel Systems). An example of an alternative, lower-cost system is the USB-9237 ( $\$ 1300$; National Instruments, Austin, TX, USA). Another option is the DT9814 (\$418; Data Translation, Marlborough, MA, USA); however, it only has one-fourth the input capacity of the former. Unfortunately, low-cost systems are compromised by low sampling rates or fewer inputs, and though it is possible to multiplex several electrodes into a single channel, this requires additional labor. Financially challenged labs can avoid such difficulties using the manual multiplexing technique we describe herein to conduct preliminary studies. We demonstrate that the DT9814, with a few accessories, is an adequate acquisition subsystem that can be assembled for $<\$ 600$.

An MEA1060-INV amplifier (\$9500, 1200 fixed gain, 1-1000 $\mathrm{Hz}$ bandwidth; Multichannel Systems) was connected to a signal divider unit (\$250; Multichannel Systems), (Figure 1). The signal divider provides easy access to all 60 amplifier outputs, but ships with just four mini-banana plug connectors (Figure 2). This allows for monitoring three or fewer channels, since one connector must serve as a ground. Relocation of connectors to different electrodes complicates the recording process. Additional connectors can be obtained, but maintaining the spatial relationship among individual channels while probing different regions becomes cumbersome, introduces delay, and creates room for error.

We therefore devised a multichannel connector to enable the simultaneous manipulation of 16 plugs without altering their spatial relationship. Additional plugs ( $<\$ 50$; Bürklin Electronics, Munich, Germany) were assembled in a plastic housing $(<\$ 10$; RadioShack, Fort Worth, TX, USA) (Figure 2). Holes were made in the plastic housing that were large enough to accommodate the plugs, but small enough to block the passage of the plug shroud (not visible), and holes were made in the metal backing plate to accommodate 26-gauge wires (Figure 2). A common ground connection for all channels at the signal divider was established by soldering 20-gauge solid wires to form a grid for attaching all of the channel grounds. (The grounding grid enabled the use of shielded or twisted-pair wire to minimize electrical noise pickup.) The connector was assembled by feeding 16 wires through openings in the grounding grid, through the backing plate hole, and soldering one wire to each of the 16 banana plugs. The tip of each plug was then inserted into one of the plastic housing holes and the entire assembly was snapped together (Figure 2). This final multichannel interface was inserted into a set of 16 sockets on the signal divider box (Figure 2).

The DT9814 has a 12-bit analogto-digital converter with a maximum sampling rate of $50,000 / \mathrm{s}$. When divided among 16 inputs, the sampling rate is reduced to $3125 / \mathrm{s}$ per channel, which allows 1-millisecond signals to be observed (3). Acquisition software was developed with the Microsoft Visual C\# 2008 Express Edition (freely available at Microsoft.com). The DT9814 data acquisition system comes with several software development libraries, including a .NET library for C\# development. The compiled program is a command-line executable that configures the 16 input channels (maximum gain 8) to utilize the dynamic range of the A/D converter over its $\sim 0.1 \mathrm{~V}$ amplitude range, gathers data for $30 \mathrm{~s}$, and finally saves the collected data as a set of 16 raw data files (2 bytes/ 12-bit sample). Recording is therefore not continuous, avoiding potential artifacts when switching locations. The 32-bit Windows .NET executable and source code utilized herein are freely available from Aragón Science (AragonScience.com). Following 
A

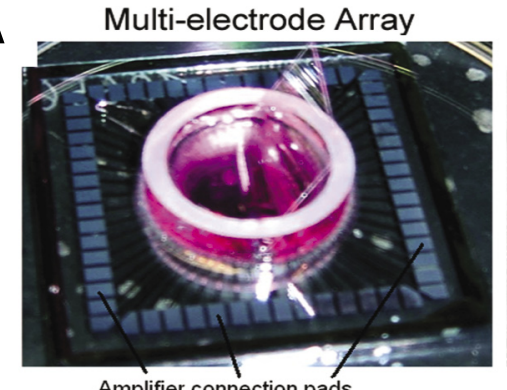

Amplifier connection pads

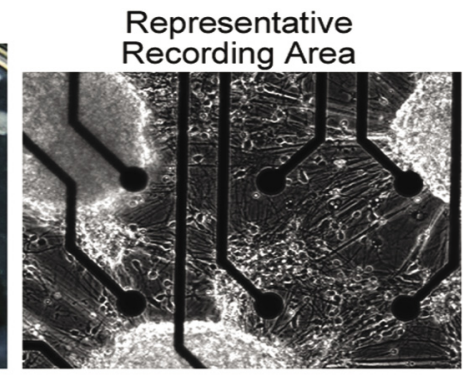

Representative

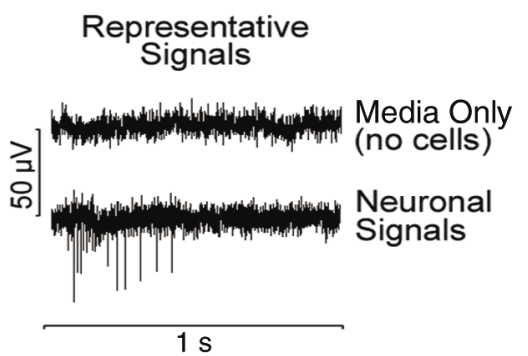

$1 \mathrm{~s}$

B

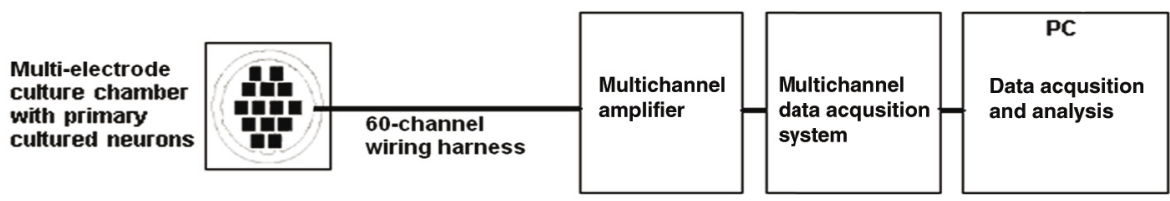

C

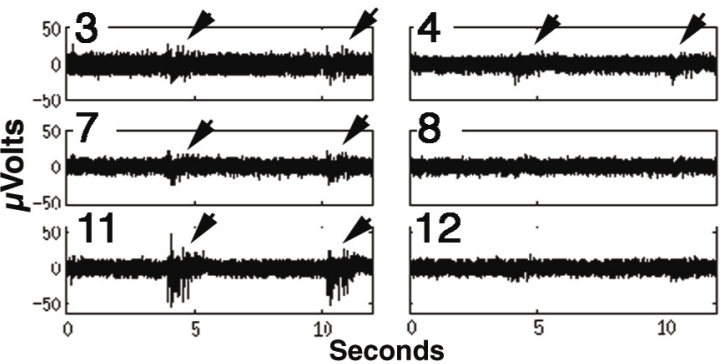

D

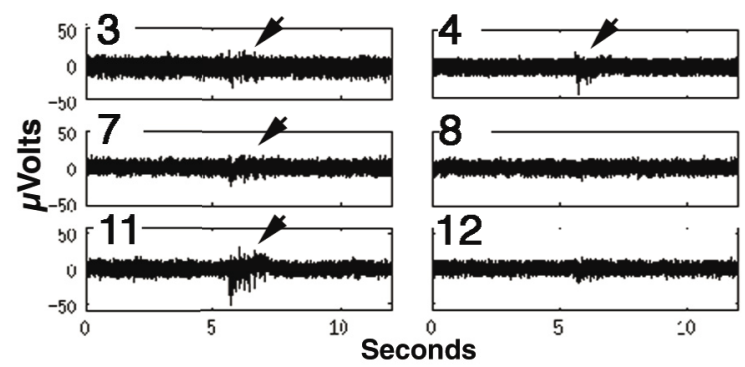

E
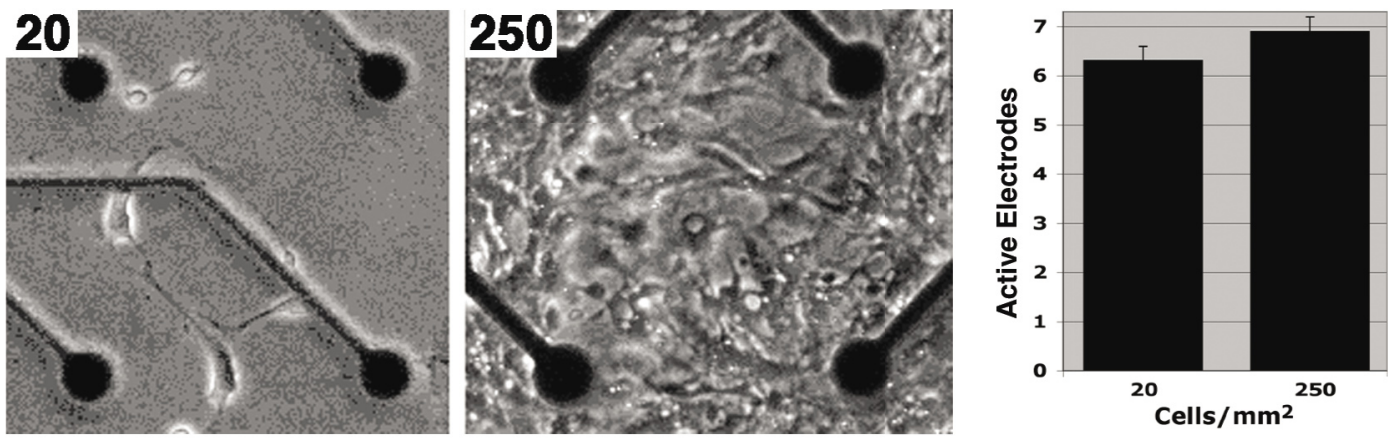

Figure 1. The multi-electrode array (MEA) and system diagram. (A, left panel) An MEA. Culture medium is within the central cell culture chamber. The chamber is permanently mounted on a glass square whose edges are lined with a total of 60 amplifier connection pads. The MEA is then placed within the MEA1060-INV (Multichannel Systems) amplifier unit (not shown), which interfaces with each MEA connection pad. (A, center panel) Sixty circular electrodes within the culture dish are connected to the MEA connection pads by insulated wires etched onto the glass. Here they are shown with embryonic cortical neurons after $14 \mathrm{~d}$ in culture; note the elaborate axonal network. Electrodes and wires appear as black dots and lines, respectively. (A, right panel) Representative signals obtained in the absence and presence of neurons. (B) General data acquisition and flow scheme. The MEA amplifier drives its output into a low-cost multiple-channel data acquisition system (DT9814; Data Translation). (C and D) Two separate sets of recordings from multiple electrodes within an MEA culture, numbered for orientation purposes. Note that simultaneous recording from 16 electrodes allows recording of spontaneous signals that radiate through large areas within the culture. (C) demonstrates two such spontaneous radiating signals (arrows), while (D) depicts one such signal (arrow) from a separate recording; note that the signals are simultaneously detected at electrodes 3, 4, 7, and 11 but not 8 and 12, indicating specificity of signal radiation (2). (E) Images of cultures less dense than those shown in (A); these cultures have an average of 20 and 250 cells $/ \mathrm{mm}^{2}$ as indicated. The accompanying graph represents of the number of active electrodes (i.e., ones from which signals were recorded) for these culture densities. Error bars, \pm SEM. 


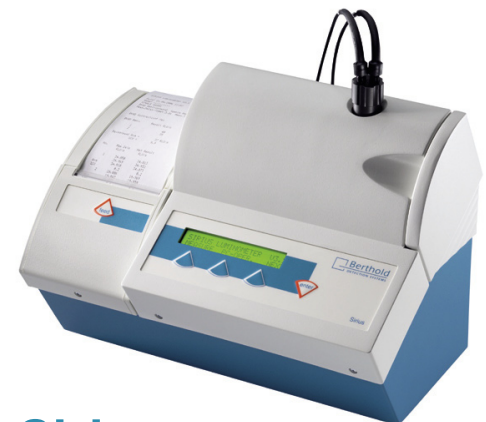

Sirius

Single Tube Luminometer 2 injectors, DLReady ${ }^{\text {TM }}$

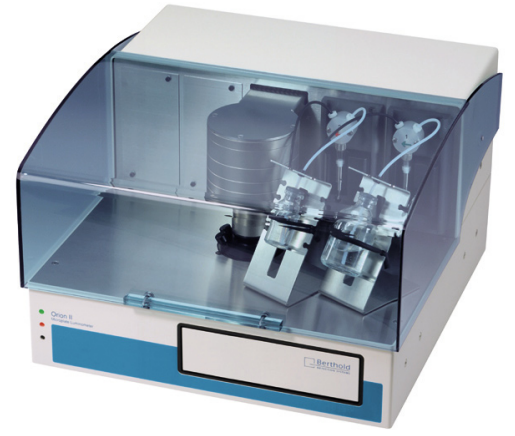

Orion II

Microplate Luminometer

4 injectors, $50^{\circ} \mathrm{C}$ incubation, 384 well, DLReady $^{\mathrm{Tm}}$

SENSITIVITY-Advanced photon counting for the best sensitivity.

FLEXIBILITY- Suitable for all known applications and easily adaptable to new requirements.

RELIABILITY - Quality workmanship, rigorous quality control, worldwide service and support.

VALUE - High quality instruments supplied in a plug-and play package, at a competitive price.

Berthold Detection Systems GmbH

Bleichstrasse 56-68, D-75173 Pforzheim Phone: $+49(0) 7231 / 9206-0$

Berthold Detection Systems USA 144 Ridgeway Square, Oak Ridge, TN 37830 Phone: 888-522-5487 (USA)

www.berthold-ds.com
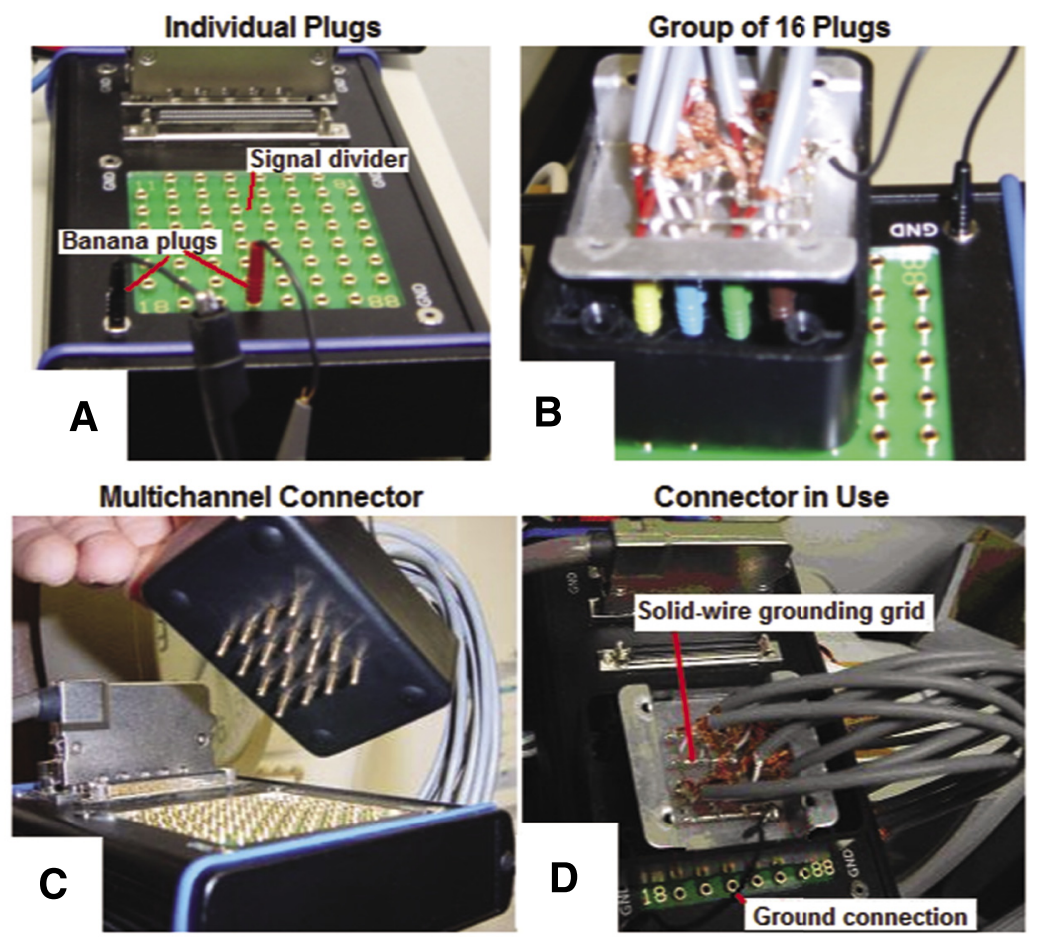

Figure 2. The multichannel connector. (A) The signal divider in normal use. Four banana plugs ship with the divider for establishing connections as shown. Note that one plug must be used for a ground connection, leaving three or fewer to probe signaling. (B) The arrangement of one of the four rows of four plugs within the multichannel connector. (C) The face of the 16-channel connector. (D) The back of the connector during use. See paragraph five for further description.

acquisition, data was processed using Matlab version R2006b. Data can only be viewed post-acquisition (a real-time visualization program is in development and will be freely available at AragonScience.com). The current software allows output as raw data and tiff files. Recording all 60 channels requires only three interruptions and relocations. But, as seen below, studies do not necessarily require analyses of the entire culture; positioning of the 16-channel acquisition interface over the optimal portion of the culture as ascertained by microscopy can minimize or eliminate the need for relocation.

Primary murine embryonic cortical neurons harvested at day 17 of gestation (C57 mice; Charles River Laboratories, Boston, MA, USA) were plated as described (4) on a standard MEA from Multichannel Systems coated with laminin (Cat. no. 23017-015; Invitrogen, Carlsbad, CA, USA) to facilitate cell attachment. Two weeks were allowed to establish an elaborate neuronal network (Figure 1) prior to recording $(2,5)$. Signals recorded with this multichannel interface (Figure 1) resemble those from prior studies $(1,2,5-7)$. While the reduced acquisition rate of this system has inherent limitations (as described in the second paragraph), we observed regional signaling of $>100 \mu \mathrm{V}$ (Figure 1A) as well as multi-site synchronous signaling as described in prior studies (Figure 1C,1D,2). Multichannel Systems specifies a maximum signal-to-noise ratio ( $\mathrm{SNR}$ ) of 14 for signals of this amplitude based on a noise level of $\pm 7.5 \mu \mathrm{V}$; we have been able to obtain noise levels $\pm 10 \mu \mathrm{V}$, yielding an SNR of 10. Addition of tetrodotoxin reversibly prevented signaling (data not shown), confirming neuronal origin (2).

We have recorded signals at multiple neuronal plating densities, and since this system uses commercial MEAs, it is also compatible with slice cultures. 


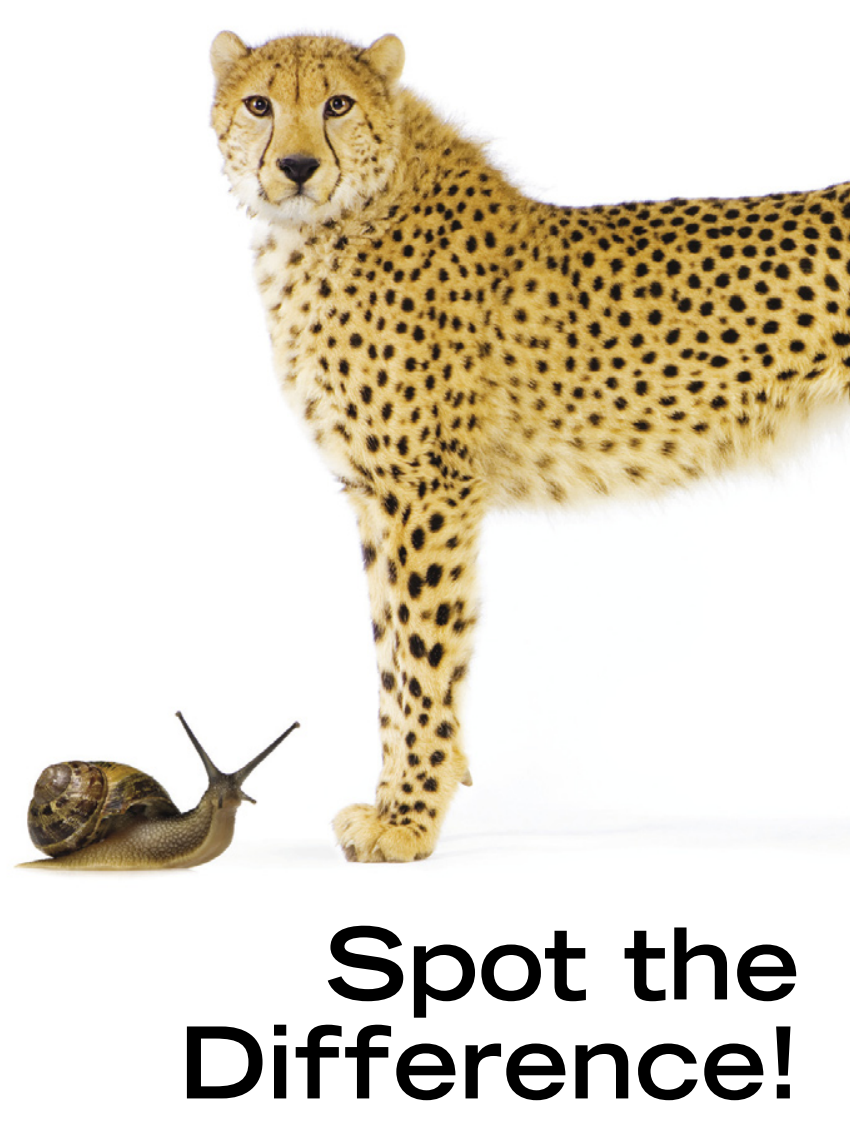

This low-cost system enables laboratories to initiate MEA-based analyses to obtain the preliminary data necessary to warrant the purchase of larger systems.

\section{ACKNOWLEDGMENTS}

The support of the US Army (contract no. W911NF06-C-0094) and helpful discussions of the research data with Dr. Elmar Schmeisser (US Army Research Office, Research Triangle Park, NC, USA) are greatly acknowledged.

\section{COMPETING INTERESTS STATEMENT}

The authors declare no competing interests.

\section{REFERENCES}

1. Wagenaar, D.A., R. Madhavan, J. Pine, and S.M. Potter. 2005. Controlling bursting in cortical cultures with closed-loop multielectrode stimulation. J. Neurosci. 25:680-688.

2. Wagenaar, D.A., J. Pine, and S.M. Potter. 2006. An extremely rich repertoire of bursting patterns during the development of cortical cultures. BMC Neurosci. 7:11.

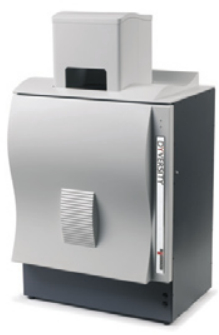

By combining Dyversity, our 16 bit CCD based 2D gel imaging system with Dymension, our rapid 2D analysis software you'll get the fastest 2D imaging and analysis ever, without compromising accuracy. So whether you want to image gels stained with Coomassie Blue, Deep Purple, Pro-Q Diamond, Silver Stain, SYPRO Ruby or Cy dyes, the only difference you'll see is how much quicker you'll get results.

To find how the
speed and
accuracy of
Dyversity and
Dymension can
help your
proteomics
research,
please

contact us.

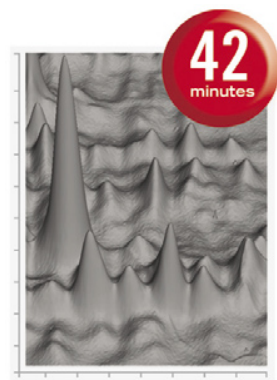

Image from Laser Scanner Scans a gel set Cy2, Cy3 and Cy5) at 100 microns.

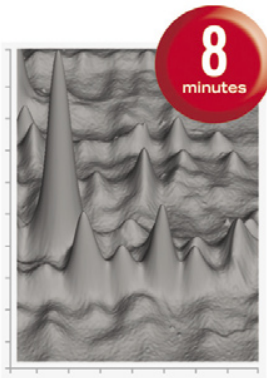

Image from Dyversity Captures a gel set (Cy2, Cy3 and Cy5) at 98 microns.

3. Black, H.S. 1953. Modulation Theory. Van Nostrand, New YorkLondon.

4. Ho, P.I., D. Ashline, S. Dhitavat, D. Ortiz, S.C. Collins, E. Rogers, and T.B. Shea. 2003. Folate deprivation induces neurodegeneration: Roles of oxidative stress and increased homocysteine. Neurobiol. Dis. 14:32-42.

5. Kamioka, H., E. Maeda, Y. Jimbo, H. Robinson, and A. Kawana. 1996. Spontaneous periodic synchronized bursting during formation of mature patterns of connections in cortical cultures. Neurosci. Lett. 206:109-112.

6. Debusk, B.C., E.J. Debruyn, J.F. Snider, J.F. Kabara, and A.B. Bonds. 1997. Stimulus-dependent modulation of spike burst length in cat striate cortical cells. J. Neurophysiol. 78:199-213.

7. Morin, F.O., Y. Takamura, and E. Tamiya. 2005. Investigating neuronal activity with planar microelectrode arrays: achievements and new perspectives. J. Biosci. Bioeng. 100:131-143.

Received 26 February 2008; accepted 10 June 2008.

Address correspondence to Thomas B. Shea, Center for Cellular Neurobiology and Neurodegeneration Research, University of Massachusetts, Lowell, MA, USA. email: thomas_shea@uml.edu

To purchase reprints of this article, contact: Reprints@BioTechniques.com

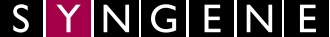<smiles>C1CCC2(CC1)CC2</smiles>

USA Tel: 800-686-4407/301-662-2863 UK Tel: +44 (0)1223 727123 